\title{
Thoroughly Testing Einstein's Special Relativity Theory, and More
}

\author{
Mario Rabinowitz \\ Armor Research, Redwood City, CA, USA \\ Email: mario715@gmail.com
}

Received 3 December 2015; accepted 18 January 2016; published 22 January 2016

Copyright (C) 2016 by author and Scientific Research Publishing Inc.

This work is licensed under the Creative Commons Attribution International License (CC BY).

http://creativecommons.org/licenses/by/4.0/

(c) () Open Access

\section{Abstract}

Einstein's Special Relativity (ESR) has enjoyed spectacular success as a mathematical construct and in terms of the experiments to which it has been subjected. Possible vulnerabilities of ESR will be explored that break the symmetry of reciprocal observations of length, time, and mass. It is shown how Newton could also have derived length contraction $L^{\prime}=L_{0} \sqrt{1-v^{2} / c^{2}}$. Einstein's General Relativity (EGR) will also be discussed occasionally such as a changed perspective on gravitational waves due to a small change in ESR. Some additional questions addressed are: Did Einstein totally eliminate the Ether? Is the physical interpretation of ESR completely correct? Why should there be a maximum speed limit, and should it always be the same? The mass-energy equation is revisited to show that in 1717 Newton could have derived the modern $m \approx m_{0} / \sqrt{1-v^{2} / c^{2}}$, and not known that it violates the foundation of his mechanics. Tributes are paid to Einstein and others.

\section{Keywords}

Vulnerabilities of Special Relativity, Challenge of Reciprocal Observations of Length and Time, Einstein, Newton, Galilean Transformation, Thought Experiments, General Relativity, Gravitational Waves

\section{Introduction}

From its inception in 1905, Einstein's Special Relativity (ESR) [1] has been the subject of much criticism [usually in error] even to the present epoch. ESR gained acceptance in America long before it was accepted in Europe. After having survived fire and anvil with spectacular success, there is no wonder criticisms are no longer tolerated. This paper will examine the theoretical foundation of ESR, and raise questions regarding traditional conclusions drawn from the allure of the equations of ESR. This paper's approach is not like any of the previous ones. It is a sympathetic position that can broaden rather than narrow ESR. 
Since experiment is the final judge and arbiter of physics, feasible thought (gedanken) experiments will be presented that examine the symmetry in ESR of reciprocal observations of length and time in which relatively moving observers each say that the other observerss length is contracted and time is slower. To my knowledge, as yet no physical experiments have been conducted to verify these predictions of ESR. My thought experiments raise questions on these issues.

Not all critics of relativity have done so in ignorance. The very core of ESR was questioned by the great physicist P. A. M. Dirac. Yet to date ESR has withstood all challenges, and stands untarnished. To my knowledge, the objections herein to the interpretation of ESR have not been previously experimentally tested or voiced, even including Paul Dirac's criticism that is worth paraphrasing and contemplating.

In some of his writings in the early 1960s, Dirac shared his thoughts regarding relativity theory [2]. He pointed out that in gravitational theory as well as in some other areas of physics the application of quantum mechanics led to an unforeseen predicament. When one looks at space-time sections (cuts) of Einstein's General Relativity (EGR), some degrees of freedom drop out of the theory. The gravitational field is a tensor field with ten components of which only 6 are needed to describe the physical world. This is the heart of the quandary. When one picks out the relevant six, this destroys the four-dimensional symmetry of space-time that was originally built into ESR and carried over into EGR. This led Dirac to question the basicness of the space-time 4-dimensional formalism of ESR in which the laws of physics must display four-dimensional symmetry. It is almost unbelievable that Dirac would come to such a conclusion, since the Dirac equation [3] for which he is the most famous, is formulated by putting space and time on an equal footing in quantum mechanics.

\section{Is the Ether (Aether) Dead or Alive?}

As early as 1679 [well before his Principia of 1687], Newton adapted the ancient Greek concept of an ether (archaic spelling is aether) as a material that is both permeable and permeates the universe for his earlier model of gravitation. In this early Newtonian model, bodies are attracted toward the earth's center because they are carried by the ether as it flows downward toward the earth's center. A decade later he introduced what has been accepted as Newton's Law of Gravitation, following priority disputes with Robert Boyle and Robert Hooke.

Subsequently, the ether was invoked as the medium of transmission for both light and gravity. For Newton although empty space is the most penetrable, it is also the hardest because the speed of light is the greatest. It was known that the harder a solid (such as diamond), the faster the speed of propagation in it. In the late 1800's, the ether was conjured as a medium that permeates all outer space, and inner space (such as between and inside atoms). The dictum was that vibrations of the ether constituted light and other electromagnetic radiation. However, the 1881 and 1887 Michelson-Morley interferometer experiments failed to detect the earth's motion through the ether. This together with ESR of 1905 seemed to deal a deathblow to the ether. But is the ether actually dead?

In 1897 Joseph Larmor [11] really wrestled with relative motion, but decided in favor of the aether: "Whatever notions may commend themselves a priori as to the impossibility of absolute space and absolute time, the fact remains that it has not been found possible to construct a system of dynamics which has respect only to the relative positions of moving bodies; and the reason suggests itself, that there is an underlying part of the phenomena, which does not usually explicitly appear in abstract material dynamics, namely, the cethereal medium...”. He had something far more significant to say about time, as noted in Section 3.1.

Einstein's General Relativity (EGR) embues empty space with properties such as curvature and metric expansion. So although he eliminated the ether and absolute space, Einstein may have inadvertently reintroduced the ether in EGR. The cosmologist E. A. Milne objected to EGR because he opposed endowing space with properties such as curvature.

By taking a small excursion into particle physics, we can see that it also imbues empty space with extraordinary properties that to some degree emulate a kind of ether and EGR. In quantum field theory, empty space is viewed as constantly forming particle-antiparticle pairs. Until they manifest themselves directly, these pairs may seem fictional and are called “virtual.” Not only can their effects be observed indirectly, but the pairs can be observed directly.

To my knowledge, the first connection between the ether of particle physics and the inadvertent ether of EGR was made by Y. B. Zeldovich. In 1967, he showed that quantum polarization of the vacuum results in a vacuum energy which has the form of Einstein's cosmological constant. In 1917, Einstein was ready to abandon the 
cosmological constant $\Lambda$. It is too weak to cause expansion between bound constituents of matter such as nuclei, molecules, and atoms. So the de facto expansion due to $\Lambda$ occurs in the space between astronomical bodies.

There is even a connection between the modern ether of particle physics and the inflationary expansion of the empty space of the early universe. First, let us step back and see how this started in a most unlikely way. In 1951, Julian Schwinger [4] reasoned that by application of a sufficiently large electric field, virtual particle-antiparticle pairs can be pulled apart before they annihilate with each other making them quite real. He calculated that the critical electric field $\boldsymbol{E}_{\text {crit }}$ at which empty space becomes unstable and effusively produces electron-positron pairs. In hindsight, this field can be obtained roughly from an elementary approach. It is that work $=$ force $\cdot$ displacement, done by $\boldsymbol{E}_{\text {crit }}$ to separate the pair a distance of the Compton wavelength $\lambda_{C}$, needs to exceed the energy of the electron-positron pair $E=2 m_{e} c^{2}$ :

$$
e \boldsymbol{E}_{\text {crit }} \lambda_{C} \geq 2 m_{e} c^{2} \rightarrow \boldsymbol{E}_{\text {crit }}>\approx \frac{2 m_{e} c^{2}}{e \lambda_{C}}=\frac{2 m_{e} c^{2}}{e\left(\hbar / m_{e} c\right)}=\frac{2 m_{e}^{2} c^{3}}{e \hbar} \sim 10^{18} \mathrm{~V} / \mathrm{m}
$$

where $m_{e}$ is the mass of the electron, $c$ is the speed of light in vacuum, $e$ is the electron charge, and $\hbar$ is the reduced Planck constant.

Equation (1) seems far removed from EGR, cosmology, and the ether. Nevertheless 20 years later in 1971, Y. B. Zeldovich and A. A. Starobinsky [5] showed that the gravitational field can play the same role as the electric field (as it did for Hawking in 1974 to get black hole radiation) [6]-[8]. If the expansion of the universe is anisotropic, empty space virtual pair production becomes a dominant factor. Surprisingly, the large production of pairs causes the universe to return to isotropy by back reaction to the stable state that we now observe.

Either by invoking the Uncertainty Principle or lack of coherence, the modern ether does not have a specific velocity associated with it on a macroscopic scale. It cannot be said to have a reference velocity such as absolute rest, with respect to which other velocities can be referred. ESR killed the old concept of the ether as a reference frame for determining absolute velocities. Neither the ether of EGR, nor the ether of vacuum spontaneous pair production can act as an absolute reference frame.

Is the ether Dead or Alive? The answer is not quite, and the present concept of a modern virtual ether does not seem to be a possible threat to ESR. The importance of acceleration, in one guise or another, should be noted in the above examples as they effect the modern or "virtual aether" which is called "space."

\section{Reciprocal Observations of Length and Time}

\subsection{Einstein's Special Relativity (ESR) Derivation}

Einstein [1] considers two systems (frames) moving uniformly with velocity $v$ relative to each other in the $x$-direction. In this idealized thought experiment, at time $t=0$ a point source of light fixed at the origin of each system emits a pulse of light when their origins coincide. Within the context of Einstein's derivation, the symmetry of Reciprocal Observations seems unassailable because the symmetry is built into the derivation. The systems are inertial relative to each other to begin with. A small acceleration of one of the frames may be needed to attain velocity $v$. Even if this was to break the symmetry (cf. Section 3.2), the theory would be oblivious to this.

It is important to bear in mind his precise words. Some of his text is put in bold font for emphasis: "In the first place it is clear that the [transformation] equations must be linear on account of the properties [assumption] of homogeneity which we attribute to space and time". A spherical light wave is at the heart of the derivation of ESR Light has a spherical wave front in the stationary unprimed system $x^{2}+y^{2}+z^{2}=c^{2} t^{2}$, where $c$ is the speed of light assumed to be invariant (constant) with respect to all inertial (constant velocity) systems. Under the assumption of homogeneity, he finds an equation of the same form in the primed moving system, $x^{\prime 2}+y^{\prime 2}+z^{\prime 2}=c^{2} t^{\prime 2}$. Here Einstein takes the step that not only space but time transforms from one system to another. He emphasizes that "our two fundamental principles are compatible", because in the beginning of his paper he introduces two postulates that he allows may be irreconcilable: "The wave under consideration is therefore no less a spherical wave with velocity of propagation $c$ when viewed in the moving system. This shows that our two fundamental principles are compatible..." "Examples of this sort, together with the unsuccessful attempts to discover any motion of the earth relatively to the "light medium," suggest that the phenomena of electrodynamics as well as of mechanics possess no properties corresponding to the idea of absolute rest. They 
suggest rather .., the same laws of electrodynamics and optics will be valid for all frames of reference for which the equations of mechanics hold good. We will raise this conjecture (the purport of which will hereafter be called the 'Principle of Relativity') to the status of a postulate, and also introduce another postulate, which is only apparently irreconcilable with the former, namely, that light is always propagated in empty space with a definite velocity $c$ which is independent of the state of motion of the emitting body.... The introduction of a 'luminiferous ether' [aether] will prove to be superfluous inasmuch as the view here to be developed will not require an "absolutely stationary space" provided with special properties, nor assign a velocityvector to a point of the empty space in which electromagnetic processes take place."

It should be noted that there is a third postulate or assumption implicit in [1]. Einstein assumes: "If a material point is at rest relatively to this system of co-ordinates, its position can be defined relatively thereto by the employment of rigid standards of measurement and the methods of Euclidean geometry, and can be expressed in Cartesian co-ordinates". As discussed in Section 8.2, a non-Euclidean geometry is required to resolve Ehrenfest's rotating disk anomaly as a precursor to EGR.

It is noteworthy that "unsuccessful attempts to discover any motion of the earth relatively to the 'light medium'" indicates that he was aware of the Michelson-Morley experiments of 1881 and 1887 [9]. He heard of it in his student days when he conceived of a similar but much less sensitive experiment using thermocouples to measure the temperature difference between light beams reflected parallel and anti-parallel to the earth's motion.

It is remarkable that although "ether' ... will not require an 'absolutely stationary space' ... nor assign a velocity-vector to a point of the empty space"” clearly dictates against the then prevailing concept of ether (aether), but leaves open the door to the modern virtual aether discussed in Section 2.

Einstein equated the two equations for the two coordinate system wave fronts [1]:

$$
x^{2}+y^{2}+z^{2}-c^{2} t^{2}=x^{\prime 2}+y^{\prime 2}+z^{\prime 2}-c^{2} t^{\prime 2}
$$

From this starting point, he goes on to derive the reciprocal observation equations for length and time where each observer says the other's rod is shorter (length contraction), and each observer says the other's clock is running slower (time dilation). This follows from his assumption that the two frames are initially in motion relative to each other. Rest frames will be designated by either a subscript 0 or no subscript, and moving frames by a prime. Subscripts 1 and 2 designate different positions $x$ and time $t$. As given below, the equations Einstein derived in this thought experiment, can be found in modern relativity textbooks.

$$
\begin{aligned}
& x_{1}^{\prime}=\frac{x_{1}-v t}{\sqrt{1-v^{2} / c^{2}}} \text { and } x_{2}^{\prime}=\frac{x_{2}-v t}{\sqrt{1-v^{2} / c^{2}}} \\
& \Rightarrow L_{0} \equiv x_{2}-x_{1}=\frac{x_{2}^{\prime}+v t^{\prime}}{\sqrt{1-v^{2} / c^{2}}}-\frac{x_{1}^{\prime}+v t^{\prime}}{\sqrt{1-v^{2} / c^{2}}}=\frac{x_{2}^{\prime}-x_{1}^{\prime}}{\sqrt{1-v^{2} / c^{2}}} \equiv \frac{L^{\prime}}{\sqrt{1-v^{2} / c^{2}}} \rightarrow L^{\prime}=L_{0} \sqrt{1-v^{2} / c^{2}} \\
& t_{1}^{\prime}=\frac{t_{1}-v x_{1} / c^{2}}{\sqrt{1-v^{2} / c^{2}}} \text { and } t_{2}^{\prime}=\frac{t_{2}-v x_{1} / c^{2}}{\sqrt{1-v^{2} / c^{2}}} \Rightarrow T^{\prime} \equiv t_{2}^{\prime}-t_{1}^{\prime}=\frac{t_{2}-v x_{1} / c^{2}}{\sqrt{1-v^{2} / c^{2}}}-\frac{t_{1}-v x_{1} / c^{2}}{\sqrt{1-v^{2} / c^{2}}} \equiv \frac{T_{0}}{\sqrt{1-v^{2} / c^{2}}} .
\end{aligned}
$$

These equations together with $y^{\prime}=y$ and $z^{\prime}=z$ derived assuming an ether by Lorentz in 1899 were named The Lorentz Transformation by Poincare in 1905. If one transforms from Frame 1 (system 1 ) to Frame 2 with a relative velocity $v_{1}$, and then Frame 3 with a relative velocity $v_{2}$, the total velocity $v_{3}$ is given by the Einstein addition of velocities:

$$
v_{3}=\frac{v_{1}+v_{2}}{1+v_{1} v_{2} / c^{2}}
$$

This guarantees that such transformations cannot exceed the speed of light $c$. To my knowledge, Equation (5) has not been experimentally verified.

In relativistic equations one finds terms like $c+v$ and $c-v$ that appear to violate Equation (5). These only have the appearance of the simple addition of velocities because of their form, but have a different origin related to path lengths as explained in Section 3.2.

Of the concepts introduced by Einstein's 1905 ESR paper, the most radical or unique concept was elimination of the ether in frames moving with constant velocity. The concepts of length contraction with velocity, and mass increase with velocity had long before been introduced. [10]. Joseph Larmor [11] appears to have antic- 
ipated time dilation in 1897 prior to Lorentz and Einstein in saying "individual electrons describe corresponding parts of their orbits in times shorter for the [rest] system in the ratio $\sqrt{1-v^{2} / c^{2}}$." Yet as late as 1927 (22 years after ESR) he argued that an absolute time was crucial to astronomy.

There is an interesting similarity between the postulates of ESR and the Cosmological Principle which existed in various forms prior to 1905 that the universe is isotropic at every point of space and is everywhere homogeneous. The steady-state model of the universe was offered in 1948 by Hermann Bondi, Thomas Gold, and Fred Hoyle as an extension of the cosmological principle to include time. It embodied the Perfect Cosmological Principle which is closer to ESR: The universe on a large scale is essentially the same in every direction, from every spot in it, and at every time. Hoyle coined the term Big Bang as a disparaging remark.

\subsection{Thought Experiments to Test Reciprocal Length and Time Observations}

Let us consider simple heuristic thought experiments using Rods and Light Beams intended to give insight into the question of reciprocal length and time observations between an inertial frame $I$ and a frame $A$ that can be accelerated. The condition $c \gg v$ will be invoked as a less stringent requirement than having $c$ constant with respect to all frames. "Rod or Line" means an object having distance between two end points that are always at rest relative to each other. This is different from, but in the same spirit as Einstein's Rods and Light in [1]. As Einstein said, everything should be made as simple as possible, but not simpler. Frame $A$ can either be observed during acceleration or as an inertial frame with constant velocity $v$. Rest frames will be designated by a subscript 0, and moving frames by a prime. As shown in Figure 1, Rod A of length $L_{A O}$ and Rod I of length $L_{I 0}$ are equal length Rods that are both initially in the same rest frame 0 . A Line of measured length $n L_{I O}$ acts as a benchmark (ruler) in the rest frame 0. A Reflecting Mirror is attached at the end of Rod A. - A Laser Light Pulse Source is positioned as shown at the left end of the rest frame system of Rods A and I and the Line.

Figure 2 shows Rod A in motion to the right with average acceleration $a$ having velocity $s+\int a \mathrm{~d} t=s+a T_{E 0}$, where $s$ is its initial speed. Rod A moves a distance $s T_{E 0}+a T_{E 0}^{2} / 2 . T_{E 0}$ is the rest frame time to traverse the

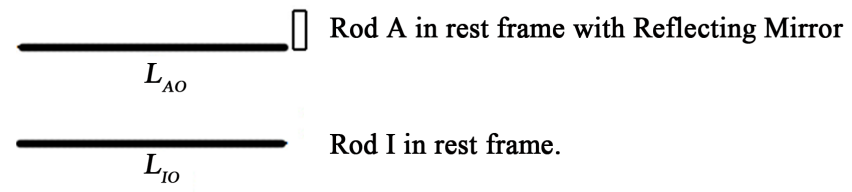

- Laser Light Pulse Source

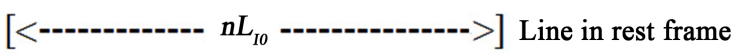

Figure 1. Rod A, Rod I, and Line of measured length $n L_{I O}$ all at rest in the same rest frame 0. A Reflecting Mirror is at the end of Rod A. • Laser Light Pulse Source is at the left end of the system.
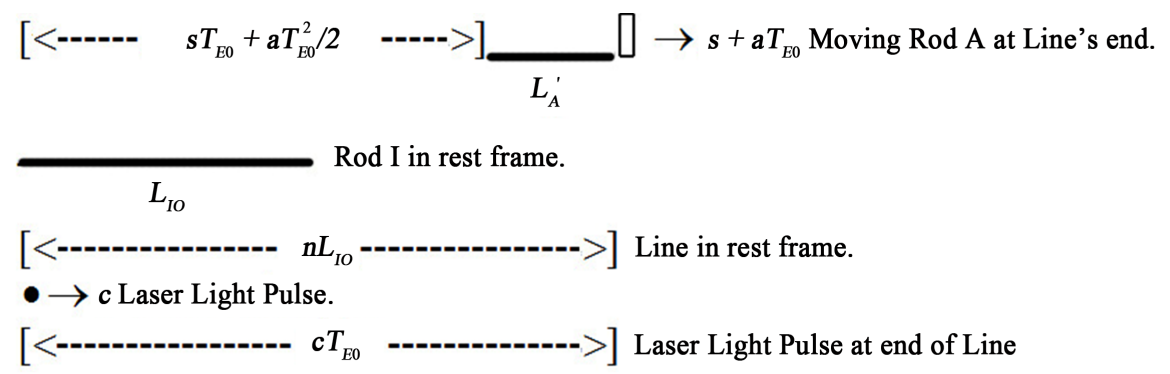

Figure 2. Rod A with attached Mirror moves at velocity $s+a T_{0 E}$ ( $s$ is the initial speed, and $a$ is the average acceleraion) to the right with respect to the rest frame of Rod I and the Line in time $T_{0 E}$ for the front of Rod A to reach the end of the Line (as measured in the Line's rest frame). In this time, the light pulse (depicted by a dot) travels a distance $s T_{0 E}+a T_{0 E}^{2} / 2$ to reach the end of the Line. 
original Emanating (Emission) direction. This motion is to the right with respect to the rest frame of Rod I and the Line $n L_{I 0}$ (measured in rest frame 0 ) for the front of Rod A to reach the end of the Line (as measured in the Line's rest frame). In this time $T_{E 0}$ a laser light pulse (depicted by a dot) with velocity of light $C$ travels a distance $c T_{E 0}$ to reach the end of the Line of length $n L_{I O}$. A Refecting Mirror (whose purpose is explained in Figure 4) is attached to the end of Rod A.

In Figure 3, let us consider Rod A to be at rest, and the Line to be moving to the left with velocity $v$ with respect to the rest frame of Rod A. When the light pulse (depicted by a dot) is at the right end of stationary Rod A, the light pulse has traveled a distance $c T_{E}^{\prime}=L_{A 0}$ in time $T_{E}^{\prime}$ as measured by a clock in the moving frame. In time $T_{E}^{\prime}$, the Line has moved to the left a distance $v T_{E}^{\prime}$.

From Figure 2 and Figure 3 the total distance traversed (path) as a sum of separate segments is utilized to relate the various variables in the two frames. In Figure 2, the path equation is:

$$
c T_{E 0}=n L_{I O}=\left[s T_{E 0}+a T_{E 0}^{2} / 2\right]+L_{A}^{\prime}=\left[v T_{E 0}\right]+L_{A}^{\prime}
$$

where to begin with for the sake of clarity and brevity, the acceleration is temporarily suspended, and $\left[s T_{E 0}+a T_{E 0}^{2} / 2\right]$ is replaced by $\left[v T_{E 0}\right]$, an inertial system with velocity $v$.

In Figure 3, the path equation is

$$
n L_{I}^{\prime}=v T_{E}^{\prime}+c T_{E}^{\prime}=v T_{E}^{\prime}+L_{I O}
$$

Thus from Equations (6) and (7)

$$
\begin{gathered}
\frac{v T_{E 0}}{c T_{E 0}}=\frac{n L_{I O}-L_{A}^{\prime}}{n L_{I O}}=\frac{v}{c} \\
\frac{v T_{E}^{\prime}}{c T_{E}^{\prime}}=\frac{n L_{I}^{\prime}-L_{0 A}}{L_{0 A}}=\frac{v}{c}
\end{gathered}
$$

Note that by Equations (8) and (9) the different times have cancelled out and subtle questions (discussed in Section 3.3) of time need not arise now in the derivation of lengths.

From Equation (8):

$$
n L_{I O}-L_{A}^{\prime}=\frac{v}{C} n L_{I O} \rightarrow L_{A}^{\prime}=n L_{I O}\left(1-\frac{v}{c}\right)
$$

From Equation (9):

$$
n L_{I}^{\prime}-L_{A 0}=\frac{v}{C} L_{A 0} \rightarrow n L_{I}^{\prime}=L_{A 0}\left(1+\frac{v}{C}\right) \rightarrow L_{A 0}=n L_{I}^{\prime} /\left(1+\frac{v}{C}\right)
$$

Dividing Equation (10) by Equation (11):

$$
\begin{aligned}
& \frac{L_{A}^{\prime}}{L_{A 0}}=\frac{n L_{I 0}\left(1-\frac{v}{c}\right)}{n L_{I}^{\prime} /\left(1+\frac{v}{c}\right)}=\frac{L_{I 0}}{L_{I}^{\prime}}\left(1-\frac{v}{c}\right)\left(1+\frac{v}{c}\right)=\frac{L_{I 0}}{L_{I}^{\prime}}\left(1-\frac{v^{2}}{c^{2}}\right) \\
& \begin{array}{l}
-\frac{\operatorname{Rod} \mathrm{A} \text { in rest frame (not moving) with Mirror. }}{L_{A O}} \operatorname{Rod} \mathrm{I} \text { in rest frame. }
\end{array} \\
& {\left[<---v T_{E}^{\prime} \quad--->\right]\left[<----c T_{E}^{\prime} \quad----->\right] \text { Light Pulse Length } c T_{E}^{\prime} \text { in moving frame B. }} \\
& -c \text { Laser Light Pulse } \\
& v \leftarrow\left[<------------n L_{I}^{\prime} \text {---------------- }>\right] \text { Line Moving to the Left relative to Rod A. }
\end{aligned}
$$

Figure 3. Rod A is at rest, and the Line is moving to the left with velocity $v$ with respect to the rest frame of Rod A. When the light pulse (depicted by a dot) is at the right end of stationary Rod A, the light pulse has traveled a distance $c T_{E}^{\prime}=L_{A 0}$ in time $T_{E}^{\prime}$ as measured by a clock in the moving frame. The Line has moved to the left a distance $v T_{E}^{\prime}$. 
Equation (12)

$$
\Rightarrow \frac{L_{A}^{\prime}}{L_{0 A}} \frac{L_{I}^{\prime}}{L_{0 I}}=\left(1-\frac{v^{2}}{c^{2}}\right)=\left(\frac{L^{\prime}}{L_{0}}\right)^{2}\left(1-\frac{v^{2}}{c^{2}}\right) \rightarrow L^{\prime}=L_{0} \sqrt{1-\frac{v^{2}}{c^{2}}}
$$

Now that we see that $n$ cancels out, and that the standard equation can be obtained, let us go back to the acceleration representation of Equation (6).

$$
\text { Equation (6) } \Rightarrow \frac{\left(s T_{E 0}+a T_{E 0}^{2} / 2\right)}{c T_{E 0}}=\frac{L_{I 0}-L_{A}^{\prime}}{L_{I 0}}=\frac{2 s+a T_{E 0}}{2 c} \rightarrow L_{A}^{\prime}=L_{I 0}\left(1-\frac{2 s+a T_{E 0}}{2 c}\right)
$$

Equation (14)/ Equation (11):

$$
\frac{L_{A}^{\prime}}{L_{A 0}} \frac{L_{I}^{\prime}}{L_{I 0}}=\left(1-\frac{2 s+a T_{E 0}}{2 c}\right)\left(1+\frac{v}{c}\right)
$$

The form of Equation (15) with $\frac{L_{A}^{\prime}}{L_{A 0}} \frac{L_{I}^{\prime}}{L_{I 0}}$ is well suited for examining possible differences in reciprocal observations of length as it gives a direct comparison within one equation.. To remind us that Equation (15) is on track, let $\frac{L_{A}^{\prime}}{L_{A 0}} \frac{L_{I}^{\prime}}{L_{I 0}} \equiv\left(\frac{L^{\prime}}{L_{0}}\right)^{2}$. Thus for no acceleration, $a=0$ and initial velocity $s=v$ in Equation (15):

$$
\begin{gathered}
\left(\frac{L^{\prime}}{L_{0}}\right)^{2}=\left(1-\frac{2 v+0}{2 c}\right)\left(1+\frac{v}{c}\right)=\left(1-\frac{v}{c}\right)\left(1+\frac{v}{c}\right)=\left(1-\frac{v^{2}}{c^{2}}\right) \\
\rightarrow L^{\prime}=L_{0}\left(1-\frac{v^{2}}{c^{2}}\right)^{1 / 2}
\end{gathered}
$$

which is the standard equation as we saw in Equation (13) and in Equation (3) Section 3.1. Now for the important task to determine if the two ratios can be unequal.

By Equation (17) for the inertial frame we can substitute $\frac{L_{I}^{\prime}}{L_{I 0}}=\left(1-\frac{v^{2}}{c^{2}}\right)^{1 / 2}$ into Equation (15):

$$
\begin{gathered}
\frac{L_{A}^{\prime}}{L_{A 0}}\left(1-\frac{v^{2}}{c^{2}}\right)^{1 / 2}=\left(1-\frac{2 s+a T_{E 0}}{2 c}\right)\left(1+\frac{v}{c}\right) \\
\rightarrow \frac{L_{A}^{\prime}}{L_{A 0}}=\left(1-\frac{2 s+a T_{E 0}}{2 c}\right) \frac{\left(1+\frac{v}{c}\right)}{\left(\left(1+\frac{v}{c}\right)\left(1-\frac{v}{c}\right)\right)^{1 / 2}}=\left(1-\frac{2 s+a T_{E 0}}{2 c}\right)\left(\frac{\left(1+\frac{v}{c}\right)}{\left(1-\frac{v}{c}\right)}\right)^{1 / 2}
\end{gathered}
$$

We require $v=s+a T_{E 0}$, as the relative velocity between Rod I and Rod A. Substituting this into Equation (18); and with $s=0$ and $v=a T_{E 0}$ :

$$
\frac{L_{A}^{\prime}}{L_{A 0}}=\left(1-\frac{0+v}{2 c}\right)\left(\frac{\left(1+\frac{v}{c}\right)}{\left(1-\frac{v}{c}\right)}\right)^{1 / 2}=\left(1-\frac{v}{2 c}\right)\left(\frac{\left(1+\frac{v}{c}\right)}{\left(1-\frac{v}{c}\right)}\right)^{1 / 2}>\left(1-\frac{v}{c}\right)\left(\frac{\left(1+\frac{v}{c}\right)}{\left(1-\frac{v}{c}\right)}\right)^{1 / 2}=\left(1-\frac{v^{2}}{c^{2}}\right)^{1 / 2}=\frac{L_{I}^{\prime}}{L_{I 0}}
$$

Equation (19)

$$
\Rightarrow \frac{L_{A}^{\prime}}{L_{A 0}}>\frac{L_{I}^{\prime}}{L_{I 0}}
$$


Thus even a small $a$ makes a difference with a surprising result that it persists even if the acceleration $\rightarrow 0$ as $v$ is approached. If so, a rod in an accelerated frame will NOT contract as much as a rod in an inertial frame that has the same velocity. The acceleration appears to break the symmetry even when it is small; and even when the acceleration ends because acceleration increased the energy state of Rod $L_{A}$. One may expect a similar inequality for times as for lengths. However, an even more glaring inequality will be analyzed in the next section.

\subsection{Time Dilation Is Not Only Relative, It Can Also Be Asymmetric}

Because the above two-Rod experiment was purposely analyzed in such a way that time cancelled out, a subtle dilemma was avoided. As we will see next, this thought experiment in which length dilation was first examined, also scrutinizes the relativity of time dilation in a way that demonstrates its asymmetry in coming and going. For the sake of clarity we will omit the effects of acceleration. This also allows the omission of the subscripts $A$ and $I$ as this is not necessary at constant velocity. At first we will pursue the original experiment, and find an anomalous asymmetry. Because of its importance, the derivation will be pared down to its essential simplest form. From the above figures and analysis, for velocity $v$ we have two traversed distance (path) equations: As can be seen from Figure 3, the path equation is:

$$
v T_{E}^{\prime}=n L^{\prime}-L_{O}=n L^{\prime}-c T_{E}^{\prime} \rightarrow T_{E}^{\prime}=\frac{n L^{\prime}-c T_{E}^{\prime}}{v}=\frac{n\left[L_{0} \sqrt{1-\frac{v^{2}}{c^{2}}}\right]-c T_{E}^{\prime}}{v} .
$$

$n L_{0}=c T_{E}$ in Equation (21):

$$
T_{E}^{\prime}=\frac{\left[c T_{E} \sqrt{1-\frac{v^{2}}{c^{2}}}\right]-c T_{E}^{\prime}}{v}
$$

$v T_{E}^{\prime}=c T_{E} \sqrt{1-\frac{v^{2}}{c^{2}}}-c T_{E}^{\prime}$ in Equation (22):

$$
T_{E}^{\prime}(v+c)=c T_{E} \sqrt{1-\frac{v^{2}}{c^{2}}}
$$

Equation (23)

$$
\Rightarrow T_{E}^{\prime}=T_{E} \frac{c}{(v+c)}\left[\frac{(c+v)^{1 / 2}(c-v)^{1 / 2}}{c}\right]=T_{E}\left[\frac{c-v}{c+v}\right]^{1 / 2}
$$

Equation (24) is anything but the standard time dilation equation. [All will turn out well in the long run.] First to be sure about Equation (24), let us see if we get the same result by using a different distance equation.

From Figure 2, we have for the path equation:

$$
\begin{gathered}
c T_{E}=v T_{E}+L^{\prime}=v T_{E}+L_{O} \sqrt{1-\frac{v^{2}}{c^{2}}} \rightarrow T_{E}(c-v)=L_{O} \sqrt{1-\frac{v^{2}}{c^{2}}}=c T_{E}^{\prime} \sqrt{1-\frac{v^{2}}{c^{2}}} \\
\left.\left.\rightarrow T_{E}(c-v)=c T_{E}^{\prime}\left[1-\frac{v^{2}}{c^{2}}\right]^{1 / 2} \rightarrow T_{E}^{\prime}=T_{E} \frac{(c-v)}{c}\right] \frac{c}{(c+v)^{1 / 2}(c-v)^{1 / 2}}\right]=T_{E}\left[\frac{(c-v)}{(c+v)^{1 / 2}(c-v)^{1 / 2}}\right] \\
\rightarrow T_{E}^{\prime}=T_{E}\left[\frac{(c-v)^{1 / 2}}{(c+v)^{1 / 2}}\right]=T_{E}\left[\frac{c-v}{c+v}\right]^{1 / 2}=T_{E} \sqrt{\frac{(c-v)}{(c+v)}}
\end{gathered}
$$

Unfortunately, this did NOT solve the dilemma, as Equations (26) and (24) are the same. So we need to look further. Note that $c+v$ and $c-v$ occur in the above equations. This is just a consequence of the summation of path length segments as seen from Equations (21), (25), and (27). Even though it looks like a simple addition of velocities it doesn't violate Einstein's addition of velocities Equation (5) of Section 3.1. 
Figure 4 shows the Light Pulse reflected to the left from the Mirror attached at the end of Rod A of rest length $L_{A 0}$, which is moving at velocity $v$ to the right. $T_{R}$ is the Return Time in the rest frame for the Light Pulse to traverse the Reverse path (Reflection path) at velocity $c$. Here the focus will be on the Return Time by means of the Return path. As before, rest frames will be designated by a subscript 0 , and moving frames by a prime.

The path equation is

$$
c T_{R}^{\prime}=L_{A 0}^{\prime}+v T_{R}^{\prime}
$$

Equation (27)

$$
\begin{gathered}
\rightarrow T_{R}^{\prime}(c-v)=L_{A 0}^{\prime} \rightarrow T_{R}^{\prime}=\frac{L_{A 0}^{\prime}}{(c-v)} \text {, Rod and Light Pulse in opposite direction. } \\
T_{E}^{\prime}=\frac{L_{A 0}^{\prime}}{(c+v)}, \text { Rod and Light Pulse in same direction. }
\end{gathered}
$$

Equations (29) + (4):

$$
\begin{aligned}
& T^{\prime}=T_{E}^{\prime}+T_{R}^{\prime}=L_{A 0}^{\prime}\left[\frac{1}{(c+v)}+\frac{1}{(c-v)}\right]=L_{A 0}^{\prime}\left[\frac{(c-v)+(c+v)}{c^{2}-v^{2}}\right]=L_{A 0}^{\prime}\left[\frac{2 c}{c^{2}-v^{2}}\right] \\
& \rightarrow T^{\prime}=L_{A 0}\left(1-\frac{v^{2}}{c^{2}}\right)^{1 / 2}\left[\frac{2 c}{c^{2}-v^{2}}\right]=L_{A 0}\left(\frac{c^{2}-v^{2}}{c^{2}}\right)^{1 / 2}\left[\frac{2 c}{c^{2}-v^{2}}\right]=L_{A 0}\left[\frac{2}{\left(c^{2}-v^{2}\right)^{1 / 2}}\right]
\end{aligned}
$$

Equation (31)

$$
\rightarrow T^{\prime}=L_{\mathrm{A} 0}\left[\frac{2}{\left(c^{2}-v^{2}\right)^{1 / 2}}\right]=\frac{2 L_{A 0}}{c\left(1-\frac{v^{2}}{c^{2}}\right)^{1 / 2}}=\frac{T_{E 0}+T_{R 0}}{\left(1-\frac{v^{2}}{c^{2}}\right)^{1 / 2}} \rightarrow T^{\prime}=T_{0}\left(1-\frac{v^{2}}{c^{2}}\right)^{-1 / 2}
$$

which is the standard Time Dilation Equation as derived by Einstein (cf. Section 3.1). The dilemma was that the time dilation for each path separately is of a different form. The two times can be different because the path lengths are different, but it is troubling that the form of time dilation differs. In some ways the experiment of Section 3.2 is a very natural thought experiment because light traverses the same frame that can be either viewed at rest or in motion. That $T_{E}^{\prime} \neq T_{R}^{\prime}$ is not surprising because of the asymmetry in path lengths. But what is surprising is that the time dilations are different! I don't think this is related to "simultaneity issues."

There is less naturalness, but symmetry, in the configuration of parallel mirrors in two relatively moving frames with light reflecting with equal angles of incidence and reflection from the relatively moving mirrors. In the common rest frame, light reflects perpendicular to the mirrors. In this symmetric configuration, $T_{E}=T_{R}$

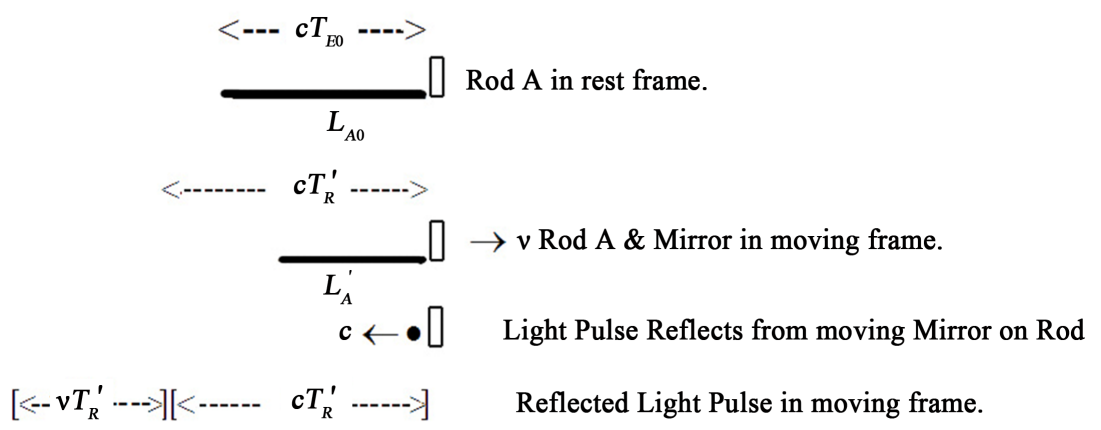

Figure 4. The Light Pulse reflects from the Mirror on Rod A of rest length $L_{A 0}$, moving at velocity $v$. $T_{R}$ is the rest frame Return Time for the Light Pulse to traverse the Reverse path. 
with $T=T_{E}+T_{R}=2 T_{E}=2 T_{R}$, and the dilation is the same in both paths for the moving frame case that has equal length diagonal paths. This is not so in the above gedanken experiment where the symmetry is broken.

It is clear that observers in different frames can observe totally different things. For example, if you drop a ball from an airplane (neglecting the atmosphere), the ball will fall straight down relative to you. However a stationary observer on the ground will see the ball in a parabolic trajectory. However, it is far less clear that time should depend on the completion of a cycle. The issue is NOT that a shorter distance yields a shorter time, but rather that the time dilation is asymmetrical (different) depending on the path taken. In ESR time and space are supposed to be symmetrically interlinked. But it appears that dilated time is not symmetrical in these thought experiments.

\section{Why Should There Be a Maximum Speed Limit?}

Special Relativity in [1] crowns the speed of light as the maximum speed in the universe with respect to length and time. Perhaps the locality of interactions may be more important. There is at least one maximum speed for the transmission of electromagnetic interactions. Einstein took it to be the speed of light. Are there different maximum speeds for different interactions just as the speed of sound is the maximum speed for acoustic interactions? Refer to Section 7 for additional insights into this question.

Of the different speeds of light, the signal velocity is considered to be the maximum speed limit of nature. Note that the subsequently added designation of "signal velocity" of light was not in the original ESR 1905 paper [1], though Einstein used this term in his recollections [12], and some limiting velocity was important in Einstein's original thinking. Though he spoke of "light signals", the term "signal velocity" was not in [1] though it was defined by Lord Rayleigh [13] in 1881. Light has four kinds of velocities of propagation [14]: group velocity, energy velocity, signal velocity, and the usually not pertinent phase velocity. All four vary strongly in a medium near an absorber's resonance frequency, where the group velocity can be significantly greater than the other three. The distinctions are moot in vacuum.

Some have thought that the Scharnhorst effect [15] may in principle contradict Special Relativity. An insight into the Scharnhorst effect relates to one of the two interpretations of the Casimir effect. One interpretation (explanation) is that because of the limited distance between two conducting plates, some virtual particles present in vacuum fluctuations have wavelengths that are too large to fit between the plates. This causes the density of virtual particles between the plates to be less than outside the plates. Hence a photon going between the plates may spend less time interacting with the decreased density of virtual particles, increasing the photon's speed extremely slightly. This is similar to the speed of light increasing as one goes from a medium like glass to vacuum because of fewer detouring interactions. The actual speed limit $c$ may be greater than its value in vacuum. So the speed of light should increase as the plate spacing approaches zero.

Milonni and Svozil [16] argue that the Scharnhorst effect cannot in principle result in "signal velocities" larger than that of light in vacuum even for two closely spaced conducting plates. They make the noteworthy observation (where I have put two sentences in bold type):

"We conclude, therefore, that no measurement of the faster-than-c velocity of light is possible. It is worth noting that our conclusion assumes the small value of the fine structure constant [alpha] determined by $e, \hbar$, and c. In a universe in which alpha were large, our conclusion would not hold. In other words, our conclusion rests on the small value of the fine structure constant rather than the basic dynamical laws of physics. This is not the first example where a violation of causality is ruled out by the values of constants rather than dynamical laws.” This was also noted by P.C.W. Davies.

Possibly a maximum speed limit may be related to a maximum acceleration limit. However a maximum time integration of a small acceleration is a different matter. In deriving length contraction and time dilation [1], Einstein purposely omitted acceleration that affects spacetime metrics [unknown in 1905]. Acceleration with concomitant gravitational time dilation is crucial in resolving the twin paradox when the twins (clocks) are finally brought together again.

Newton was concerned with the infinite speed of propagation in his Gravitational Theory. One of his concerns was that with an infinite velocity, events in the distant universe can have an immediate chaotic effect locally. And there is a quantum kind of disorder on a micro scale.

A Galilean transformation is essential in Newton's Absolute Space and Time for Newton's Mechanics. For reasons related to kinetic energy given in the next Section 5, Newton was unaware of this. It is noteworthy that a 
finite maximum velocity yields a Lorentz transformation; and that an infinite maximum velocity yields a Galilean transformation. This can be seen heuristically from Einstein's Equations (3) and (4) in Section 3.1:

$$
x^{\prime}=\frac{x-v t}{\sqrt{1-v^{2} / c^{2}}} \underset{c \rightarrow \infty}{\longrightarrow} x-v t \text { and } t^{\prime}=\frac{t-v x_{1} / c^{2}}{\sqrt{1-v^{2} / c^{2}}} \underset{c \rightarrow \infty}{\longrightarrow} t .
$$

\section{Mass-Energy Variation with Velocity}

\subsection{Clarification and Deeper Insight}

In my last paper [10], there was a lesson to be learned that needs to be emphasized. The equation for the relativistic mass variation with velocity $m(v)$ can be derived for an accelerating mass. Although the derivation differs from the traditional relativistic approaches for an inertial mass, the result is identical. An analogous argument can be made for momentum $p(v)$.

The relation between energy and work done $\mathrm{d} E=F \cdot \mathrm{d} x=F \cdot v \mathrm{~d} t$ or equivalently power $P=\mathrm{d} E / \mathrm{d} t=F \cdot v$, with the force, $F=\mathrm{d}(m v) / \mathrm{d} t$ can be considered fundamental. This is the starting approach in [10] and in [17] for the analysis of the radiation reaction force in which Max Abraham and his contemporaries in the 1800's were referenced as taking a similar approach. In the modern era, this power equation was given relativistically in 1950 by Goldstein [18], but no analysis was presented that the relativistic mass variation with velocity $m_{\text {acc }}=m_{0} / \sqrt{1-v^{2} / c^{2}}$ can be derived from it for either an inertial or an accelerating mass. I have not seen this done elsewhere.

Reference [10] presented two derivations in parallel. This was done for brevity that sequential derivations could not provide. Each derivation is general in one way, but not in another. The Newtonian derivation is general in that it involves no electrodynamics, nor Einstein's postulates. However the Newtonian derivation is limited to $v \ll c$. The more interesting case (and also the more controversial as shown in Sections 5.2 and 5.3) is the Newtonian derivation.

The non-Newtonian derivation is general in that it does not require $v \ll c$, but it does use Einstein's postulate that $\mathrm{c}$ is constant with respect to all frames. It yields the novel result that the energy-mass relation holds in all frames i.e. even accelerating frames with any time rate of change of acceleration. Let us first look at this case [10] whose only connection to electrodynamics is the use of $E \approx m c^{2}$ as given by numerous well-known papers of the 1800's.

The overall derivation is short and straightforward. So that the reader need not be troubled by having to obtain the original paper and then find the appropriate section, here is a summary of the relevant steps from Equations (7) and (8) in reference [10]. The numerical factor $f \sim 1$.

$$
\begin{aligned}
\frac{\mathrm{d} E}{\mathrm{~d} t}= & F \cdot v \rightarrow \frac{\mathrm{d}\left(f m c^{2}\right)}{\mathrm{d} t}=\left[m \frac{\mathrm{d} v}{\mathrm{~d} t}+v \frac{\mathrm{d} m}{\mathrm{~d} t}\right] v \rightarrow f c^{2} \frac{\mathrm{d} m}{\mathrm{~d} t}=m v \frac{\mathrm{d} v}{\mathrm{~d} t}+v^{2} \frac{\mathrm{d} m}{\mathrm{~d} t} \\
& \rightarrow \int_{m_{0}}^{m} \frac{\mathrm{d} m}{m}=\int_{v_{0}}^{v} \frac{v \mathrm{~d} v}{\left(f c^{2}-v^{2}\right)} \rightarrow \ln \left(\frac{m}{m_{0}}\right)=\frac{-1}{2}\left\{\ln \left[1-\frac{v^{2}}{f c^{2}}\right]\right\}
\end{aligned}
$$

With $v_{0}=0 \Rightarrow m_{0}$ is the rest mass in the rest frame. So Equation (33) $\Rightarrow$

$$
m_{a c c} \equiv m=\frac{m_{0}}{\sqrt{1-v^{2} / f c^{2}}}
$$

and with $f=1$ :

$$
E=m_{a c c} c^{2} \equiv m c^{2}=\frac{m_{0} c^{2}}{\sqrt{1-v^{2} / c^{2}}}
$$

which is the standard equation. However, notably in this derivation, the velocity $v$ can be time varying, since there was no restriction that the velocity $v$ is constant as for an inertial frame. Although Equations (34) and (35) are identical to Einstein's Special Relativity equation after [1], (he then used a different derivation), it opens the possibility of a new interpretation. That $m(v)$ holds for an accelerating mass can be significant in fields as re- 
mote as Gravitational Radiation as discussed in Section 6. Many different analyses have similar starting points. What is important are the differences in the end points. Because of acceleration, my derivation of $m=m_{0} / \sqrt{1-v^{2} / c^{2}}$ is not amenable to the symmetrical reciprocal observation interpretation that needs to be experimentally tested.

\subsection{Could Newton Really Have Gotten the Modern Mass-Energy Equation?}

The concept of energy does not occur in Isaac Newton's writings. Yet he was clearly aware of the energy concept because he was keenly aware of Gottfried Leibniz' writings. Newton probably ignored energy because his rival Leibniz championed it. Newton's Principia was full of Geometrical Calculus, although he was fully adept at Differenial Calculus which was lacking in Leibniz' Calculus.

Interestingly for Leibniz, Kinetic Energy $=m_{0} v^{2}$ rather than the present day $1 / 2 m_{0} v^{2}$. He called it vis viva. If Newton used Leibniz' Kinetic Energy for Newton's light corpuscles (particles) going at velocity $c$, he very likely would have started the analysis with $\mathrm{d}\left(m c^{2}\right) / \mathrm{d} t$ with $c=$ the speed of light. This could have led him to the mass-energy relation, and as a bonus the modern day Kinetic Energy $=1 / 2 m_{0} v^{2}$ as shown by the binomial expansion of Equation (10) in [10]. As presented by proposing that matter and light are interconvertible, and with the approximation $v \ll c$, in 1717 Newton could not only have derived

$$
E=m_{a c c} c^{2} \equiv m c^{2} \approx \frac{m_{0} c^{2}}{\sqrt{1-v^{2} / c^{2}}}
$$

he could have deduced that the corpuscles of light going at speed c must have rest mass $m_{0}=0$. This is because, as seen from Equation (36), $v=c$ leads to the unphysical $E=\infty$ unless $m_{0}=0$.

Since no modern physics was used in Section 3.2 as discussed in Section 7, Newton could also have derived length contraction $L^{\prime}=L_{0} \sqrt{1-v^{2} / c^{2}}$ which should have caused him to question the Galilean Transformation. But it is unlikely that he would have derived time dilation because of his strong bias for absolute time.

So far Newton has been given much deserved credit and everything looks rosy, except for a fly in the ointment as analyzed next in Section 5.3.

\subsection{Mass Variation Results in a Galilean Transformation Dilemma}

As we shall see, a variable mass is not compatible with a Galilean Transformation and hence absolute spacetime. Though likely not for Newton, this may have been a possible deterrent to early acceptance of mass variation with velocity. This is a problem even with a Newtonian derivation with $v \ll c$.

Let us bear in mind that a conserved quantity is a quantity that does not change over time. An invariant quantity is a quantity that does not depend on the frame of reference. When a frame of reference is changed, energy and momentum (which are non-invariant) change. However, they remain conserved within each frame of reference. For example a body may have both kinetic energy and momentum in a moving frame, but both these conserved quantities of a body are 0 in the body's rest frame. The incompatibility of mass variation with velocity for a Galilean Transformation is known to specialists in the field. Here is how the analysis goes.

\section{A. Elastic Collisions \& Galilean Frames in Same Direction}

$$
\begin{aligned}
& \text { Frame I Mass } m_{2} \text { is stationary } \\
& \text { Before } \\
& \stackrel{\bullet v_{1}}{m_{1}} \quad \stackrel{\bullet}{m_{2}} \rightarrow 0 \\
& \text { After } \\
& \stackrel{\bullet}{m_{1}} \stackrel{v_{1}}{m_{2}} \rightarrow v_{2} \\
& v_{b}^{2}=v_{1}^{2}+\frac{m_{2}}{m_{1}} v_{2}^{2}
\end{aligned}
$$

Frame II

$$
\begin{aligned}
& \text { Before After } \\
& \text { - } \rightarrow v_{b}-v \quad-v \leftarrow \bullet \\
& m_{1} \quad m_{2} \\
& \stackrel{\bullet}{m_{1}} \stackrel{v_{1}}{m_{2}} \stackrel{\bullet}{\rightarrow} v_{2}-v
\end{aligned}
$$


Conservation of K.E. in II:

$$
\begin{aligned}
& \frac{1}{2} m_{1}\left(v_{b}-v\right)^{2}+\frac{1}{2} m_{2}(-v)^{2}=\frac{1}{2} m_{1}\left(v_{1}-v\right)^{2}+\frac{1}{2} m_{2}\left(v_{2}-v\right)^{2} \\
& \text { Equation (38) } \rightarrow\left(v_{b}-v\right)^{2}+\frac{m_{2}}{m_{1}}(-v)^{2}=\left(v_{1}-v\right)^{2}+\frac{m_{2}}{m_{1}}\left(v_{2}-v\right)^{2}
\end{aligned}
$$

Equation (39) $\rightarrow v_{b}^{2}-2 v_{b} v+v^{2}+\frac{m_{2}}{m_{1}} v^{2}=v_{1}^{2}-2 v_{1} v+v^{2}+\frac{m_{2}}{m_{1}} v_{2}^{2}-2 \frac{m_{2}}{m_{1}} v_{2} v+\frac{m_{2}}{m_{1}} v^{2}$

$$
\begin{aligned}
& \text { Equation (40) } \rightarrow v_{b}^{2}-2 v_{b} v=v_{1}^{2}-2 v_{1} v+\frac{m_{2}}{m_{1}} v_{2}^{2}-2 \frac{m_{2}}{m_{1}} v_{2} v \\
& \text { Equation (41) } \rightarrow v_{b}^{2}=v_{1}^{2}+\frac{m_{2}}{m_{1}} v_{2}^{2}+2 v\left(v_{b}-v_{1}-\frac{m_{2}}{m_{1}} v_{2}\right)
\end{aligned}
$$

Conservation of Momentum in Frame I: $m_{1} v_{b}+m_{2}(0)=m_{1} v_{1}+m_{2} v_{2}$.

$$
\text { Equation (43) } \rightarrow v_{b}=v_{1}+\frac{m_{2}}{m_{1}} v_{2} \rightarrow v_{b}-v_{1}-\frac{m_{2}}{m_{1}} v_{2}=0
$$

Equation (44) in (42)

$$
\Rightarrow v_{b}^{2}=v_{1}^{2}+\frac{m_{2}}{m_{1}} v_{2}^{2}+2 v(0)=v_{1}^{2}+\frac{m_{2}}{m_{1}} v_{2}^{2} \text {, i.e. } v_{b}^{2}=v_{1}^{2}+\frac{m_{2}}{m_{1}} v_{2}^{2}=\text { Equations (37) and }
$$

$\Rightarrow$ If Momentum is Conserved in Frame I, then KE is conserved in all Frames, provided $m_{2} / m_{1}$ remains constant.

In Special Relativity the mass

$$
m=m_{0} / \sqrt{1-v^{2} / c^{2}}
$$

Equation (46) in (43):

$$
v_{b}-v_{1}-\frac{m_{2}}{m_{1}} v_{2}=v_{b}-v_{1}-v_{2}\left[\frac{m_{02}}{m_{01}}\right]\left[\frac{1-\frac{v_{2}^{2}}{c^{2}}}{1-\frac{v_{b}^{2}}{c^{2}}}\right]^{1 / 2}=v_{b}-v_{1}-v_{2}\left[\frac{m_{02}}{m_{01}}\right]
$$

only for $v_{2}= \pm v_{b}$, or for $c \rightarrow \infty$. So, Equation (47) does not hold in general.

B. Elastic Collisions \& Galilean Frames in Different Directions [Velocity Components]

As before from Conservation of Kinetic Energy in Frame I:

$$
v_{b}^{2}=v_{1}^{2}+\frac{m_{2}}{m_{1}} v_{2}^{2}
$$

As before from Conservation of K.E. in Frame II:

$$
v_{b}^{2}=v_{1}^{2}+\frac{m_{2}}{m_{1}} v_{2}^{2}+2 v\left(v_{b}-v_{1}-\frac{m_{2}}{m_{1}} v_{2}\right)
$$

Conservation of Momentum in Frame I:

$$
m_{1} v_{b}+m_{2}(0)=m_{1} v_{1}+m_{2} v_{2}
$$

Equation (50)

$$
\rightarrow v_{b}=v_{1}+\frac{m_{2}}{m_{1}} v_{2} \rightarrow v_{b}-v_{1}-\frac{m_{2}}{m_{1}} v_{2}=0
$$


Equation (51) in Equation (38) gives Equation (37). So if Kinetic Eenergy is conserved in Frame I, it is conserved in Frame II. The conclusion still holds that variable mass is incompatible with a Galilean Transformation. Note that Conservation of Momentum was used in Frame II, so $v=v_{x}+v_{y}$ can be anything.

Because of his apparent disinterest in kinetic energy, it seems likely that Newton did not know that variable mass is incompatible with a Galilean Transformation. Therefore as shown in [10], Newton could very well have derived the equation $m \approx m_{0} / \sqrt{1-v^{2} / c^{2}}$ and not have been at all aware that it violates the foundation of his mechanics--the Galilean Transformation [Newton's equation of motion is used differently and properly for time varying mass where constituents are included. This ranges from the motion of rockets and meteorites which lose mass, to planets which gain mass by accretion of surrounding matter].

\section{Accelerating Mass and Gravitational Radiation}

A small paradigm shift can make a big difference. Now that we have seen (cf. Section 5) that $m=m_{0} / \sqrt{1-v^{2} / c^{2}}$ holds even for accelerating masses, we can apply this fact in situations where this can make a difference such as expectations regarding gravitational radiation (waves). Conventional analysis can be found in [19]. Let us see how a change in perspective in ESR can influence the analysis and conclusion in EGR since there is a synergism between the two.

When it comes to the acceleration of mass, one has to be cautious about subtleties that are often only obvious in hindsight. Contrary to conventional wisdom, there may be gravitational dipole radiation, when time changing mass is taken into account Let us start with a simple example that there is neither monopole gravitational radiation, nor electromagnetic monopole radiation. This becomes clear when we realize that monopole radiation means that the source and the field must be spherically symmetric. In both the electric and gravitational cases, the source can maintain monopole symmetry by accelerating radially in and/or out in what may be called the breathing mode. A Gaussian surface around-and external to-the radially symmetric (radially expanding and/or contracting) body shows that the field acts as if the source remains unmoved at the center of spherical symmetry. The field outside the Gaussian surface is static and therefore not radiating. In the electromagnetic case, the absence of a non-symmetry implies there is no magnetic field and only an electric field. Hence the Poynting vector-which is necessary for electromagnetic radiation-is zero.

A common misconception is that gravitational radiation is only quadrupole because there are no gravitational dipoles. Since mass appears to be only attractive, some mistaking conclude that there are no gravitational dipoles. Nevertheless, there are gravitational dipoles $D_{G}$ just as electric dipoles can be formed from only one sign of charge.

$$
D_{G}=\sum_{i} m_{i} r_{i} \Rightarrow \frac{\mathrm{d} D_{G}}{\mathrm{~d} t}=\sum_{i} m_{i} \frac{\mathrm{d} r_{i}}{\mathrm{~d} t}+r_{i} \frac{\mathrm{d} m_{i}}{\mathrm{~d} t}=\sum_{i} m_{i} \dot{r}_{i}+r_{i} \dot{m}_{i}
$$

The conventional approach takes $\dot{m}_{i}=0$ :

$$
\Rightarrow D_{G}=\sum_{i} m_{i} v_{i}
$$

For radiation we need acceleration, and for the conventional $\dot{m}_{i}=0$ :

$$
\therefore \frac{\mathrm{d}^{2} D_{G}}{\mathrm{~d} t^{2}}=\sum_{i} m_{i} \ddot{r}=\sum_{i} m_{i} \dot{v}_{i}=0 \text {, conventionally. }
$$

Equation (54) says that conventionally there is no gravitational dipole radiation when there is no driving force, which is tantamount to the conservation of linear momentum in this case. Similarly, there is no gravitational analog of electromagnetic dipole radiation which results from acceleration $\mathrm{d}^{2} / \mathrm{d} t^{2}$ of the magnetic dipole moment. The gravitational analog is mass angular momentum $L$, where $\mathrm{d} L / \mathrm{d} t=0$ by conservation of angular momentum; and hence $\mathrm{d}^{2} L / \mathrm{d} t^{2}=0$.

However, when $\dot{m}_{i} \neq 0$, Equation (52) becomes

$$
D_{G}=\sum_{i} m_{i} \dot{r}_{i}+r_{i} \dot{m}_{i} \Rightarrow \frac{\mathrm{d}^{2} D_{G}}{\mathrm{~d} t^{2}}=\sum_{i} m_{i} \ddot{r}+r_{i} \ddot{m}_{i}=\sum_{i} m_{i} \dot{v}_{i}+r_{i} \ddot{m}_{i} \Rightarrow \frac{\mathrm{d}^{2} D_{G}}{\mathrm{~d} t^{2}}=\sum_{i} m_{i} \ddot{r}+r_{i} \ddot{m}_{i} \neq 0 \text { ordinarily }
$$

Thus if gravitational radiation exists, dipole radiation is not totally ruled out if $\dot{m}_{i} \neq 0$. When $\dot{m}_{i}=0$ (and 
hence $\ddot{m}_{i}=0$ ) conservation of momentum of an isolated system precludes gravitational dipole radiation, but this is not obvious if $\dot{m}_{i} \neq 0$, and hence $\ddot{m}_{i} \neq 0$ for $\dot{m}_{i} \neq$ constant. Also, if the dipole mass(es) radiate, energy and mass leaves the system, making it non-closed. In Newtonian Gravity (NG), an external force might be used to produce gravitational dipole radiation by acceleration of mass dipoles; and an external torque produce dipole radiation by angular acceleration. GR should agree with NG at low fields, aside from the speed of propagation.

After substantial expenditure of resources over decades with ever larger detection apparatus, to date gravitational waves (radiation) have not been detected directly. They were inferred from 1974 binary star orbital measurements and analysis made by 1993 Nobel Prize winners Hulse and Taylor. Using the moon as a detector, Friedwardt Winterberg [20] has proposed what may turn out to be the largest detector, and perhaps one of the least expensive ones. However since general relativity conceives of gravity in the context of geodesic motion in curved spacetime, the existence of gravitational radiation may nevertheless be questioned.

\section{Thoughts on the Length and Time Thought Experiments}

One can usually find sayings that contradict each other like "Two heads are better than one" and "Too many cooks spoil the broth.” To make them relevant to what I am about to say we could modify these aphorisms to: Two thoughts are better than one and Too many thoughts spoil the Gedanken experiment. I have included this additional perspective on the Thought Experiments of Sections 3.2 and 3.3 for a broader outlook, and in hopes that the former adage applies rather than the latter. These additional thoughts relate only to length and time presently as mass appears to be a separate question.

Note that no properties of light, or that the speed of light $c$ is a constant relative to all frames needed to be invoked. To a good approximation the requirement $c \gg \gg v$ would have sufficed [Similarly for two spherical wave fronts from two relatively very slowly moving sources using a medium other than light]. We still could have obtained the standard relativistic equations for length and time given by Equations (17) and (32):

$$
L^{\prime} \approx L_{0}\left(1-\frac{v^{2}}{C^{2}}\right)^{1 / 2} \quad(17) \text { and } T^{\prime} \approx T_{0}\left(1-\frac{v^{2}}{C^{2}}\right)^{-1 / 2} \quad \text { (32), where } C \text { is a slower observational (signal) velocity }
$$

with $C \gg \gg>C \gg>v$. The mirror could be replaced by another kind of reflecting detector. And if $C \gg \gg \gg \gg>v$, Equations (17) and (32) would reduce from a Lorentz transformation to the Galilean expressions $L^{\prime} \approx L_{0}$ and $T^{\prime} \approx T_{0}$. Or if there should be an observational speed $C>C$, the length and time equations of ESR would also readily be obtained.

The above should shed light on the observations regarding a Maximum Speed Limit in Section 4; and especially on the Thought Experiments of Section 3 themselves. It may be possible to do real physical experiments by using light to provide a meta-velocity to ascertain if Reciprocal Observations are symmetrical, with each observer saying your yardstick is shorter than mine and your clock is slower than mine. The answer lies in the details. If one has to go to a velocity $v \approx C$ in order to be able to detect the length contraction or time dilation, then this would violate the requirement that $C \gg>v$.

I am optimistic that cosmic censorship will not enter in to keep us from finding the right zone for conducting such experiments. In any event with a metavelocity role for $c \gg>C$, then real physical experiments on Reciprocal Observations should be feasible for length and time. The question of Mass Reciprocal Observations (where each observer says my mass is less than your mass was not part of Einstein's derivation in [1]) has been presently deferred for a number of reasons including violation of the Galilean Transformation. Even so, as derived in [10] and shown in Section 5.3 for $c>>v$ one may obtain for accelerating mass $m_{\text {acc }} \approx m_{0} / \sqrt{1-v^{2} / c^{2}}$ directly from Newton's second law of motion. This does not lend itself to a symmetrical reciprocal observation interpretation. Reciprocal observations of length and time are equally unphysical as those of mass. Furthermore no matter how large the contraction, ESR requires an instantaneous expansion by the same amount when an observation is made in the rest frame.

Note that Einstein's derivation of $L$ and $T$ [1] locks in inertial frames and the speed of light in the first step that is detailed in Section 3.1. He starts with two inertial frames moving relative to each other at velocity $v$ with light sources at their origins. Each emits a light pulse when their origins coincide. This results in two spherical wave fronts moving at the speed of light $c$, that he relates from his postulate that $c$ is constant with respect to all inertial frames. It is no wonder then that $c$ and inertial frames play a dominant role in his theory. This radicalizes the thought that another velocity $C$ can lead to similar equations for $L$ and $T$ as discussed immediately above. 
A legitimate question may be raised that is relevant to Einstein's abundant use of Rods [1] and the different use of Rods in this paper in Sections 3.2 and 3.3. Fundamentally Rods would need to arise from a theory of particles and/or fields, rather than be "given" phenomenological objects as they were with Einstein [1] and here. However, this is not likely to change the outcome because it is difficult to distinguish the rod becoming Lorentz-contracted for kinematic reasons (cf. Sections 8.1 and 8.2) and a physical dynamic contraction of the rod. Lorentz took the dynamic view and ultimately acquiesced to Einstein's kinematic view, nonexistence of an ether, and the equivalence of all inertial frames. Although the Lorentz equations are the same as ESR, they yield different predictions because of their disparate physical interpretation. The Lorentz Transformation (LT) argues against reciprocal observations with LT being one way from the preferred frame to any inertial frame. Nevertheless, the derivations for length, time, and mass in Sections 3.2, 3.3, and 5.1 favor ESR as they invoke no ether. Their methodology is rudimentary as they replicate both the equations and interpretation of ESR without being restricted to light as the only limiting speed.

Unlike persistent acceleration in the Ehrenfest rotating disk paradox to be discussed in the next Section 8.1, the effect of breaking the symmetry of reciprocal observations of length (Section 3.2), remains after the acceleration ends. If the rods are determined to be the same length when both of them are at rest, the rod that was accelerated goes to a higher energy state; and the system has more entropy even when the acceleration stops. The approach of path conservation in a given frame used here is quite basic.

\section{Discussion}

\subsection{A Tribute to Einstein and Others}

The ancient Greek historian Herodotus said that those who do not know history are destined (doomed) to repeat the mistakes of the past. Regrettably, most scientists are too burdened to delve into the development of theory and experimentation in their own fields. As a result they are unaware of concepts that have long ago been lost in the dustbin of history either through passive neglect or active banishment.

This year marks the $100^{\text {th }}$ Anniversary of Einstein's General Relativity (EGR) theory, which was an outgrowth of Einstein's Special Relativity (ESR) theory. ESR is a vital ingredient of EGR. The following tribute to Einstein gives insight into the development of ESR and EGR, as well as illustrating his humanity and the generous way in which he gave credit to others.

In 1922, seventeen years after introducing his theory of Special Relativity, Einstein gave a lecture in Kyoto, Japan entitled: How I Created the Theory of Relativity [12]. It is interesting to note that because he honored his commitment to talk in Japan prior to learning that he had won the Nobel Prize (for his explanation of the photoelectric effect), he forewent going to the Nobel ceremonies in Stockholm, Sweden.

"By chance a friend of mine in Bern (Michele Besso) helped me out. It was a beautiful day when I visited him with this problem. I started the conversation with him in the following way: 'Recently I have been working on a difficult problem. Today I come here to battle against that problem with you.' We discussed every aspect of this problem. Then suddenly I understood where the key to this problem lay. Next day I came back to him again and said to him, without even saying hello, 'Thank you. I've completely solved the problem.' An analysis of the concept of time was my solution. Time cannot be absolutely defined, and there is an inseparable relation between time and signal velocity. With this new concept, I could resolve all the difficulties completely for the first time."

There was a kind acknowledgement to Besso in Einstein's 1905 paper [1] that launched ESR.

Einstein was similarly gracious to his friend Marcel Grossman, who helped him with EGR [12]: "When I came back to Zurich from Prague, my friend the mathematician Marcel Grossman was waiting for me. He had helped me before in supplying me with mathematical literature when I was working at the patent office in Bern and had some difficulties in obtaining mathematical articles. First he taught me the work of Curbastro Gregorio Ricci and later the work of Riemann. I discussed with him whether the problem could be solved using Riemann theory, in other words, by using the concept of the invariance of line elements. We wrote a paper on this subject in 1913, although we could not obtain the correct equations for gravity. I studied Riemann's equations further only to find many reasons why the desired results could not be attained in this way."

Einstein was a monumental thinker and his great successes in the field of physics are unparalleled. In my opinion, his creation/realization of The Theory of General Relativity was the most profound theoretical concept achieved by the mind of man. Some of the things it includes are:

- The Equivalence Principle with the verified prediction of the bending of starlight. 
- Space-Time Curvature as the most accurate verified representation of gravity in the Universe.

- Gravitational Time Dilation, a verified correction for the GPS (Global Positioning Satelites).

- Space-Time Warping and Frame Dragging as verified by the Stanford Gravity Probe B experiment.

- Gravitational Lensing as widely verified by stellar images.

Einstein's achievements throughout physics are legion of which the above and the following are only a small number. Not well-known is his concept of Thermal Wavelength which presciently anticipated the de Broglie wavelength. This is probably why he championed the latter concept in Louis de Broglie's $1924 \mathrm{PhD}$ thesis when the physics faculty was of a mind to reject it. In low temperature physics, Einstein easily solved a problem that stumped the finest minds by replacing a vexing integral with an equivalent series. His paper with Podolsky and Rosen (known as the EPR paradox) initiated the concept and field of entanglement.

Einstein received the 1921 Nobel prize for "his services to Theoretical Physics, and especially for his discovery of the law of the photoelectric effect". His explanation of the Photoelectric Effect in which he introduced the concept of the Photon was one of his lesser theoretical achievements. The Nobel committee eschewed EGR because they seemed to misunderstand it.

Einstein's friend Paul Ehrenfest deserves homage for his penetrating insight that challenged ESR [21]. He considered a rotating disk and concluded that the circumference must contract because its motion is parallel to the velocity, but not the radius because it is perpendicular to the motion. This implies that Pi decreases, as it does for circles (except for great circles) on spaces such as a sphere, which are not Euclidean. This anomaly leads to a conflicting contradiction that Pi increases in that small measuring rods aligned around the circumference of the circle would shrink requiring more rods. By this measure the circumference increases while the radius remains unchanged. Rotation involves acceleration. This is likely what got Einstein thinking about a change in metric in EGR that accompanies acceleration. Previous to this, Einstein had the ingenious insight that acceleration is locally equivalent to a gravitational field. In 1998 Friedwardt Winterberg [22] presented a resolution of Ehrenfest's Paradox using a dynamic approach in contrast to previous kinematic interpretations. His is in the spirit of Lorentz' approach.

Special recognition is also due to the unheralded Professors David Finkelstein, Anton Lampa, Roy Weinstein, James Terrell, Paul Kard, and Oleg Jefimenko for their incisive insights into relativistic subtleties [23]-[28].

\subsection{Early Insights into Time and Space}

Einstein acknowledged the influence on some of his abstractions by the $17^{\text {th }}$ century philosopher Baruch Spinoza who opposed the concept of absolute time. In various venues [12] [29] Einstein expressed his insight into the nature of space-time:

"I wished to show that space-time is not necessarily something to which one can ascribe a separate existence, independently of the actual objects of physical reality. Physical objects are not in space, but these objects are spatially extended. In this way the concept "empty space" loses its meaning."

Einstein may have been influenced by Gottfried Leibniz' concept of space. From correspondence with Samuel Clark [probably acting as a surrogate for Newton], it is clear that Leibniz disagreed with Newton's concept of absolute space as a fixed arena for objects. For Leibniz ca. 1710, the location of an object is not a property of an independent space, but a property of the object itself together with every other object relative to it. This is almost the same as Einstein's view.

\section{Conclusions}

Einstein's Special Relativity (ESR) was derived by a thought experiment [1] and two (effectively three) postulates regarding two spherical light waves simultaneously emitted from two relatively moving frames at the instant when the two sources coincided; as well as with rods. In the present paper, by means of thought experiments with rods and a reference line, a different conclusion was reached with respect to reciprocal observations. ESR theory has been challenged in the interpretation of reciprocal observations of length and time; as well as whether other velocities can replace $c$ in the equations of length contraction and time dilation. To my knowledge, actual experiments of this kind have not yet been conducted. Experiment must decide which view is correct. My paper raises the question: Is the physical interpretation of ESR completely correct? The meaning of Einstein's postulate that physical laws are the same in all inertial frames is not clear when reciprocal observations seem contradictory. 
Section 3.2 shows that a rod whose energy state has been increased by acceleration to a velocity $v$ does not contract the same as an identical rod that remains at rest when reciprocal observations are made. Section 3.3 shows that time dilation differs depending on whether a light beam moves parallel or anti-parallel to the motion of a rod. In EGR a time delay due to the presence of the sun's gravitational field was confirmed by Irwin Shapiro in 1964 by bouncing radar signals off of Mercury and Venus. This is a different kind of asymmetry than analyzed in the present paper, but it demonstrates that it is not obvious what is pertinent and what is incidental. Without EGR, the sun's presence would have been considered incidental.

Some relativistic effects have the appearance of optical illusions similar to the optical bending of a rod placed in water due to the difference in indices of refraction [different speeds of light] in air and in water. As Lampa [24], Weinstein [25] and Terrell [26] independently demonstrated starting in 1924, a relativistic rod can look shorter, longer, and/or rotated depending on how fast it is moving, whether it is approaching, receding, or passing, and how far away it is from the observer. In 1958, Finkelstein [23] showed that the gravitational field of a point particle was not time reversal invariant. In 1997, Jefimenko [28] derived the equations of relativistic electrodynamics just by using retardation. Retardation takes into account delayed effects due to the finite speed of light. Thus for Jefimenko, there is no real length contraction. Thought experiments regarding length contraction generally boil down to questions of simultaneity. Perhaps similarly, experimentation or otherwise [such as with my thought experiments] will show that there are no real contradictory reciprocal observations where each observer says the other's rod is shorter and time is slower.

In the same spirit that Einstein and his friend Besso battled the concept of time (cf. Section 8.1), we should question and experimentally test these very anti-intuitive aspects of ESR, even though they may be part and parcel of what we think are symmetries in nature. I think it is as important to test Reciprocal Observations as it has been to try to confirm the existence of Gravitational Waves. Symmetry breaking of reciprocal observations is almost as radical a concept as time dilation when Einstein surmised it in 1905. In physics, experiment is first and foremost in deciding the correctness of theory.

To my knowledge, the length and time experimental tests I propose in Sections 3.2 and 3.3 have not been done to date. Pairs of rods, clocks, and masses need to be compared when initially in the same rest frame, and then observed after one of each is accelerated to a relative velocity, i.e. a relatively higher energy state. Section 3.2 demonstrates that length observations can be non-reciprocal when consideration is given as to which identical rod changes its energy state. Even though time and space are relative, time like space should increase uniformly in inertial frames. Nevertheless as shown in Section 3.3, time does not increase uniformly in a moving frame as it dilates differently for motion parallel to a light beam than for motion that is anti-parallel. Although experiment is often unavoidably theory laden, experiment must be the final arbiter. It may not be that hard to test Reciprocal Times. Global Positioning Sattelite (GPS) clocks in accelerating satellites signal to earth. They could signal to each other to ascertain if each says the others' time is slower. The complication that GPS also has an EGR time correction can be eliminated by temporarily turning it off.

\section{Acknowledgements}

I am grateful to David Gahagen for his steadfast assistance over decades with figures, and for patiently listening to my evolving concepts. Dr. Eduardo Cantoral's encouragement and kindly bringing various papers to my attention over the years is appreciated. Thanks are also due to Dr. Velvel Hushwater and others for their thoughtful criticisms and complements with respect to reference [10].

\section{References}

[1] Einstein, A. (1905) Annalen der Physik, 322, 891-921. http://dx.doi.org/10.1002/andp.19053221004

[2] Dirac, P.A.M. (1962) Reviews of Modern Physics, 34, 592. http://dx.doi.org/10.1103/RevModPhys.34.592

[3] Dirac, P.A.M. (1928) Proceedings of the Royal Society of London A, 117, 610-662. http://dx.doi.org/10.1098/rspa.1928.0023

[4] Schwinger, J. (1951) APS Journals Archive, 82, 664-679. http://dx.doi.org/10.1103/PhysRev.82.664

[5] Zeldovich, Y.B. and Starobinsky, A.A. (1971) Zh. Eksp. Teor. Fiz., 61, 2161.

[6] Hawking, S.W. (1975) Communications in Mathematical Physics, 43, 199-220. http://dx.doi.org/10.1007/BF02345020

[7] Rabinowitz, M. (2005) Black Hole Paradoxes. Nova Science Publishers, New York. http://arxiv.org/abs/astro-ph/0412101 
[8] Rabinowitz, M. (2006) International Journal of Theoretical Physics, 45, 877-884. https://www.researchgate.net/publication/2173131_Black_Hole_Radiation_and_Volume_Statistical_Entropy

[9] Michelson, A.A. and Morley, E.W. (1887) American Journal of Science, 34, 333-345. http://dx.doi.org/10.2475/ajs.s3-34.203.333

[10] Rabinowitz, M. (2015) Journal of Modern Physics, 6, 1243-1248. http://dx.doi.org/10.4236/jmp.2015.69129

[11] Larmor, J. (1897) Philosophical Transactions of the Royal Society A, 190, 205-300. http://dx.doi.org/10.1098/rsta.1897.0020

[12] Einstein, A. (1982) Physics Today, 35, 45-47.

[13] Rayleigh, L. (1881) Nature, 24, 382-383. http://dx.doi.org/10.1038/024382a0

[14] Panofsky, W.K.H. and Phillips, M. (1955) Classical Electricity and Magnetism. Addison-Wesley Publishing Co., Reading, MA.

[15] Scharnhorst, K. (1990) Physics Letters B, 236, 354-359. http://dx.doi.org/10.1016/0370-2693(90)90997-K

[16] Milonni, P.W. and Svozil, K. (1990) Physics Letters B, 248, 437-438. http://dx.doi.org/10.1016/0370-2693(90)90317-Y

[17] Rabinowitz, M. (2014) Advanced Studies in Theoretical Physics, 8, 1165-1176. http://dx.doi.org/10.12988/astp.2014.411142

[18] Goldstein, H. (1950) Classical Mechanics. Addison-Wesley Pub. Co., Cambridge, MA.

[19] Misner, C.W., Thorne, K.S. and Wheeler, J.A. (1971) Gravitation. Freeman \& Co., San Francisco.

[20] Winterberg, F. (2015) Zeitschrift für Naturforschung, 70a, 545-551. http://arxiv.org/abs/1503.03003

[21] Ehrenfest, P. (1909) Physikalische Zeitschrift, 10, 918.

[22] Winterberg, F. (1998) Zeitschrift für Naturforschung, 53a, 751-754.

[23] Finkelstein, D. (1958) Physical Review, 110, 965-967. http://dx.doi.org/10.1103/PhysRev.110.965

[24] Lampa, A. (1924) Zeitschrift fur Physik, 27, 138-148. http://dx.doi.org/10.1007/BF01328021

[25] Weinstein, R. (1960) American Journal of Physics, 28, 607-610. http://dx.doi.org/10.1119/1.1935916

[26] Terrell, J. (1959) Physical Review, 116, 1043. http://dx.doi.org/10.1103/PhysRev.116.1041

[27] Kard, P.A. (1961) Collection of Electrodyn. \& Special Relativity Probs. Tartu State Univ., Tartu.

[28] Jefimenko, O.D. (1997) Electromagnetic Retardation and Theory of Relativity. Electret Scientific Co, Star City.

[29] Einstein, A. (1961) Relativity: The Special and the General Theory. Wings Books, New York. 